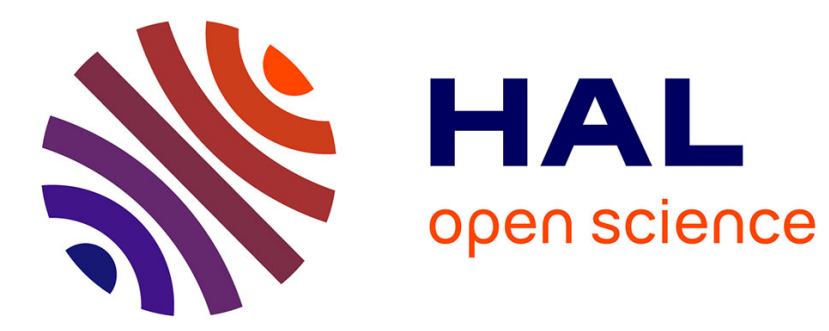

\title{
Evanescent-wave tuning of a locally resonant sonic crystal
}

Tingting Wang, Yan-Feng Wang, Yue - Sheng Wang, Vincent Laude

\section{To cite this version:}

Tingting Wang, Yan-Feng Wang, Yue - Sheng Wang, Vincent Laude. Evanescent-wave tuning of a locally resonant sonic crystal. Applied Physics Letters, 2018, 113 (23), pp.231901 (4). hal-02131444

\section{HAL Id: hal-02131444 \\ https://hal.science/hal-02131444}

Submitted on 16 May 2019

HAL is a multi-disciplinary open access archive for the deposit and dissemination of scientific research documents, whether they are published or not. The documents may come from teaching and research institutions in France or abroad, or from public or private research centers.
L'archive ouverte pluridisciplinaire HAL, est destinée au dépôt et à la diffusion de documents scientifiques de niveau recherche, publiés ou non, émanant des établissements d'enseignement et de recherche français ou étrangers, des laboratoires publics ou privés. 


\section{Evanescent-wave tuning of a locally-resonant sonic crystal}

Ting-Ting Wang, ${ }^{1,2}$ Yan-Feng Wang, 1,3, a) Yue-Sheng Wang, ${ }^{1,3}$ and Vincent Laude ${ }^{2, \text { b) }}$

${ }^{1)}$ Institute of Engineering Mechanics, Beijing Jiaotong University, 100044 Beijing, China

${ }^{2)}$ Institut FEMTO-ST, Université Bourgogne Franche-Comté and CNRS, 25030 Besançon, France

${ }^{3)}$ School of Mechanical Engineering, Tianjin University, 300350 Tianjin, China

Locally-resonant sonic crystals can support band gaps at low frequencies defined by resonances internal to the unit cell. Band gap frequencies are dictated by the choice of resonators and their interaction with the medium supporting acoustic wave propagation. We show that locally-resonant band gaps can be tuned by engineering the dispersion of the evanescent waves appearing in the propagation medium at the resonator sites. Specifically, we consider experimentally a tubular waveguide filled with different levels of water and grafted with a periodic array of acoustic resonators. Water filling continuously tunes the dispersion of evanescent waves by changing the waveguide cross-section. Dispersion relations and transmission properties are obtained with a three-dimensional time-harmonic finite element model of wave propagation. Numerical and experimental results are found to be in good agreement. The present work is relevant to the practical design of tunable acoustic devices.

During the last 15 years, a great deal of efforts has been made to achieve control of waves propagating within phononic/sonic crystals exhibiting elastic/acoustic band gaps $^{1}$. The propagation of waves is completely forbidden in the band gaps ${ }^{2}$. It has since then been observed that band gaps can be arise because of two different mechanisms, Bragg interference ${ }^{3}$ and local resonances of substructures in the unit cell ${ }^{4}$. Bragg band gaps can be generated when the wavelength has a definite relation to the periodicity. In contrast, locally resonant (LR) band gaps appear around local resonances and do almost not depend on periodicity. At resonance, the energy of waves propagating in the matrix can be efficiently stored and delayed. For a given periodicity, LR gaps can appear at wavelengths much larger than the lattice constant. $\mathrm{Nu}-$ merous applications have been researched based on locally resonant structures in the low-frequency range, such as filters ${ }^{5}$, waveguides ${ }^{6}$, negative effective parameters ${ }^{7}$, interior noise reduction ${ }^{8}$, or vibration control $^{9}$.

Because of these features of LR band gaps, various LR structures are designed and fabricated ${ }^{10,11}$. However, topology or geometrical parameters ${ }^{12-14}$ of these systems are hardly tunable. In contrast, reconfigurability is easier to realize by using sonic crystals ${ }^{15}$. Elford et al. ${ }^{16}$ designed novel noise barriers by using Matryoshka sonic crystals with different number of C-shaped shells. Wang et al. ${ }^{17}$ investigated longitudinal near-field coupling between acoustic resonators grafted along a waveguide through altering the length of resonators. Arguably, the structures proposed in the above works are hardly continuously tunable. Selectively filling a fluid into resonators has been proposed as a way to control local resonators ${ }^{18}$, but it could be difficult to control precisely and to equalize the filling level of a large number of periodic resonators in practical applications. Conse-

\footnotetext{
a) Electronic mail: wangyanfeng@bjtu.edu.cn

b) Electronic mail: vincent.laude@femto-st.fr
}

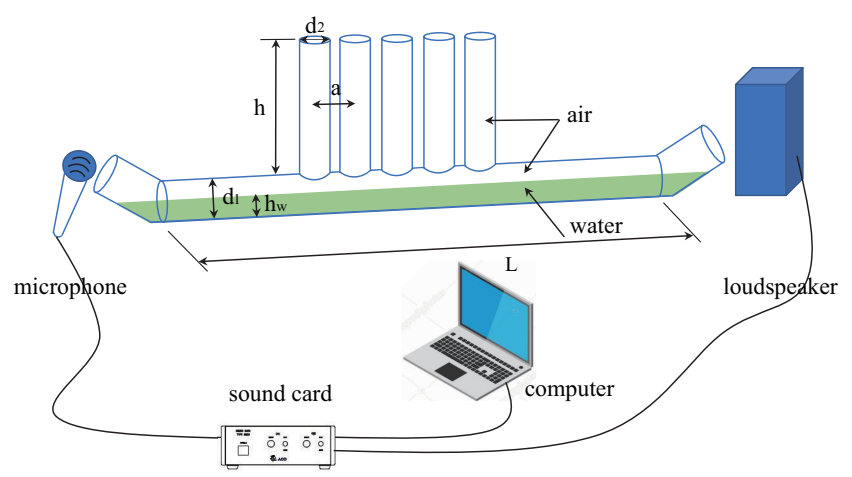

FIG. 1. Schematic drawing of the experimental setup. Transmission of sound through the sonic crystal sample is obtained using a microphone on the one side and a loudspeaker on the other side. The crystal is composed of a sequence of five tubes grafted onto a cylindrical waveguide. he white and green parts in the system represent air and water respectively. The lattice constant is $a$. Dimensions in the experiment are $L=2$ $\mathrm{m}, a=8 \mathrm{~cm}, d_{1}=10 \mathrm{~cm}, d_{2}=2.5 \mathrm{~cm}$, and $h=24 \mathrm{~cm}$. The height of water $h_{w}$ is continuously variable.

quently, changing wave propagation properties in the matrix rather than in the resonators could be an interesting alternative to obtain tunability of band gaps.

In this Letter, we consider a locally-resonant sonic crystal built using a tubular waveguide grafted with periodic acoustic resonators. Local resonance band gaps appear close to resonance frequencies and depend on the grafting conditions. Tunability is experimentally realized by filling the waveguide with water up to a controlled level, without acting on the properties of the resonators. The water level changes the waveguide crosssection and as a result tunes the dispersion of evanescent guided waves. In the following, we examine the modifications of spectral transmission that result and show tunability of locally-resonant band gaps. 


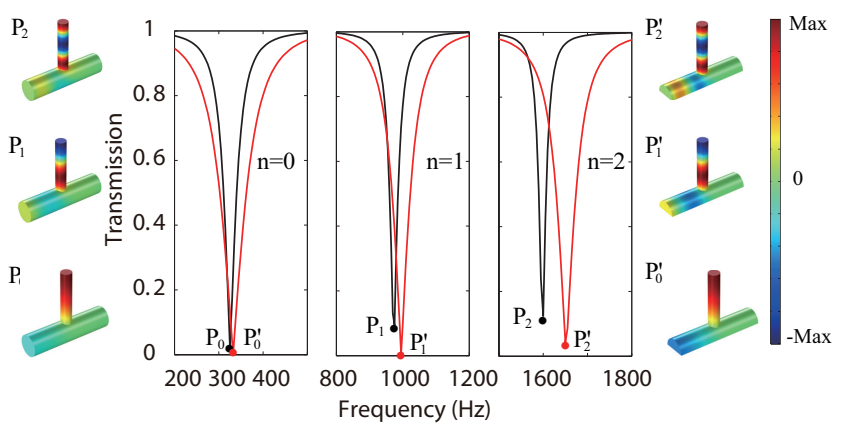

(b)

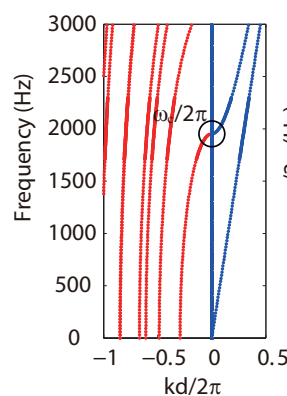

(a)

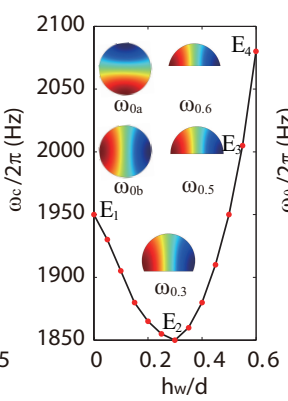

(b)

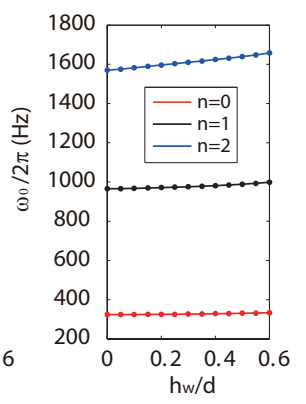

(c)

FIG. 3. Dispersion relation of the waveguide. (a) The complex band structure for guided modes is shown for half-water $\left(h_{w}=d / 2\right)$. Both the real (blue) and imaginary (red) parts of the complex dispersion relation are presented. The cut off frequency $\omega_{c} /(2 \pi)$ of the least evanescent wave is marked by the black circle. (b) $\omega_{c} /(2 \pi)$ is depicted as a function of the normalized height of water $h_{w} / d$. The insets show the pressure distribution of the least evanescent wave at cut-off for $h_{w}=0\left(\mathrm{E}_{1}\right), 0.3 d\left(\mathrm{E}_{2}\right), 0.5 d\left(\mathrm{E}_{3}\right)$ and $0.6 d\left(\mathrm{E}_{4}\right)$. The color scale is similar to that in Fig. 2. (c) The first three locally resonant frequencies for a single resonator are presented as a function of the normalized height of water.

crystal sample is constructed on a cylindrical polyvinyl chloride (PVC) tube used as waveguide for acoustic waves in air. The tube has a length $L=2 \mathrm{~m}$, an inner diameter $d_{1}=10 \mathrm{~cm}$ and a $2 \mathrm{~mm}$ thick wall. Two bent tubes are glued at the ends of the waveguide in order to hold water inside. The height of water, $h_{w}$, can be continuously adjusted by filling or removing water. A periodic array of resonators is introduced in the form of PVC tubes closed at one extremity and grafted onto the waveguide with a period $a=8 \mathrm{~cm}$. The radius of the resonators is $d_{2}=2.5 \mathrm{~cm}$, and their length $h=24 \mathrm{~cm}$. A source and a receiver for sound in the audible range are used to measure acoustic transmission through the locally-resonant sonic crystal, following the method of Ref. ${ }^{19}$.

In order to analyze experimental results, we use a time-harmonic finite element model (FEM) of pressure wave propagation $^{19}$. The sound velocity inside the tube, $c=333 \mathrm{~m} / \mathrm{s}^{-1}$, was determined experimentally and is used without any adjustment in numerical simulations. The FEM model allows us for evaluating the transmission coefficient through the waveguide. The surface separating water and air is considered as imposing a perfectly rigid boundary condition, i.e. acoustic wave propagation in water is neglected. In addition, the dispersion of propagating and evanescent guided waves is determined by computing the complex band structure ${ }^{19-21}$.

We first investigate numerically the local resonance mechanism for a single grafted resonator as a function of water height. Figure 2 shows the transmission around the three resonances that appear below $1800 \mathrm{~Hz}$. When the waveguide is empty $\left(h_{w}=0\right)$, the transmission dips appear around 327.3, 975.4 and $1601.3 \mathrm{~Hz}$. When the waveguide is half filled $\left(h_{w}=d / 2\right)$, the dips are moved upward to 332.4, 995.2, and $1651.7 \mathrm{~Hz}$. Figure 2 shows that the pressure distributions inside the resonator at the

frequencies of the dips are unaffected by the water level and correspond to the first three natural vibration modes of the resonator. The main change for the pressure distribution occurs inside the waveguide as the water level is changed.

The theoretical model of Ref. ${ }^{19}$ explains the transmission dip appearing near a resonance frequency by the coupling between evanescent guided waves originating from the grafting point and propagating guided waves. In particular, in case frequencies remain below the cut-off of the second guided mode, the center frequency of the dip is approximated by formula

$$
\omega_{0}^{2}=\Omega^{2}+\frac{\kappa_{22}}{2 \alpha},
$$

where $\Omega$ is the natural frequency of the resonator, $\alpha$ is the imaginary part of the wavenumber for the least evanescent guided mode (the wave with the smallest imaginary part of the propagation constant), and $\kappa_{22}$ is a coupling coefficient proportional to the overlap integral of the modal distributions of the least evanescent guided wave and the fundamental propagating guided wave. Loosely speaking, Equation (1) implies that energy at resonance is the sum of the energy in the resonator plus the energy of the evanescent wave. As the local resonance frequency increases, the energy in the evanescent wave must increase, since $\Omega$ remains fixed.

These observations point at the change of the dispersion relation for guided waves as the water level changes. Fig. 3(a) show the complex dispersion relation in the case that $h_{w}=d / 2$. At low frequencies, there is a single propagating guided wave with linear dispersion and speed of sound $c$. All other guided waves are evanescent, as indicated by their pure imaginary wavenumbers. 


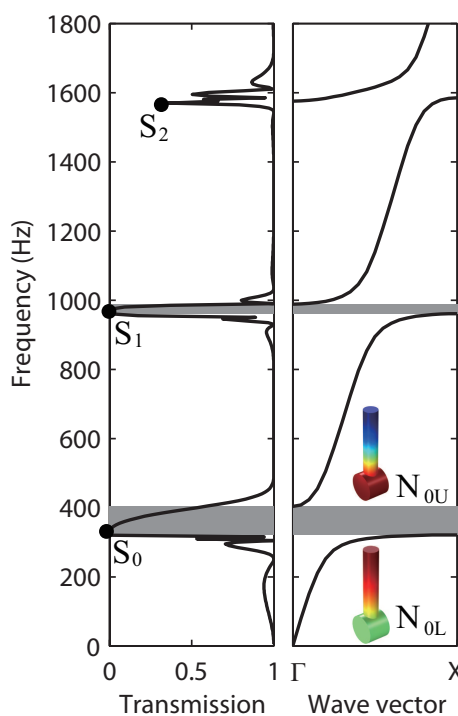

$\left(a_{2}\right)$

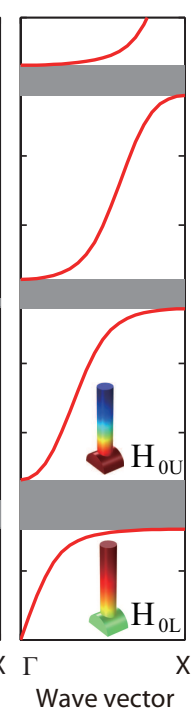

$\left(b_{1}\right)$

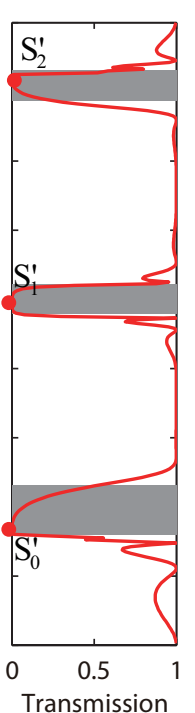

$\left(b_{2}\right)$

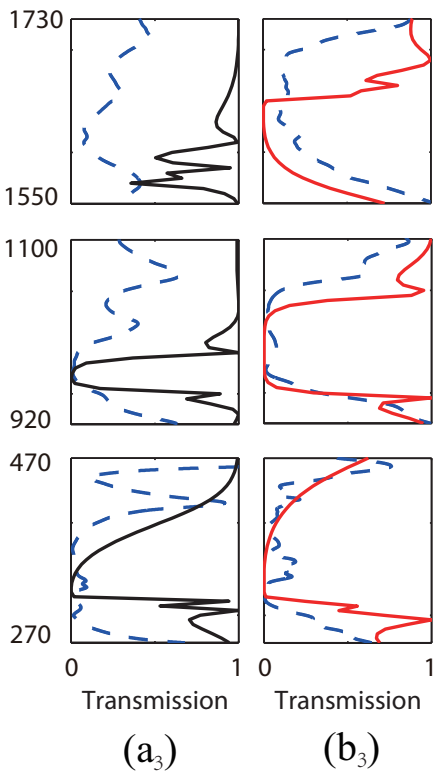

$\left(b_{3}\right)$
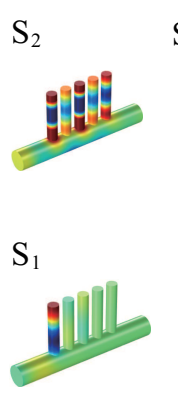

$\mathrm{S}_{0}$

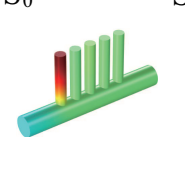

$\left(a_{4}\right)$
$\mathrm{S}_{2}^{\prime}$

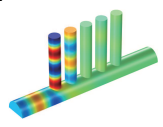

$\mathrm{S}_{1}^{\prime}$

$S_{1}^{\prime}$

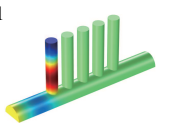

$\mathrm{S}_{0}^{\prime}$

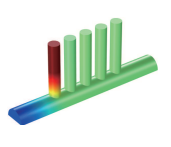

$\left(b_{4}\right)$

FIG. 4. Band structure and transmission through the sonic crystal. Band structures are shown $\left(\mathrm{a}_{1}\right.$ without $\left(h_{w}=0\right)$ and $\left(\mathrm{b}_{1}\right)$ with half-water $\left(h_{w}=d / 2\right)$. The insets show the pressure distributions of the eigenmodes at the edges of the lowest bandgap. Numerical transmission spectra are shown in $\left(a_{2}\right)$ and $\left(b_{2}\right)$, respectively. $\left(a_{3}-b_{3}\right)$ Close-up views of the numerical (solid line) and of the experimental transmission (blue dash line) around the locally resonant frequencies are plotted. (a $\left.-\mathrm{b}_{4}\right) \mathrm{Pressure}$ distributions at the 0th, 1st, and 2nd local resonance frequencies are depicted. The color scale is similar to that in Fig. 2.

The least evanescent of these waves reaches a cut-off at $\omega_{c} /(2 \pi)$, above which it becomes propagating and thus propagation is not monomodal anymore. As a note, the dispersion relation is

$$
\omega^{2}=c^{2} k^{2}+\omega_{c}^{2}
$$

for all waves, with $k$ the complex wavenumber, and only the value of $\omega_{c}$ is different between different waves. Fig. $3(\mathrm{~b})$ shows the variation of the cut off frequency of the least evanescent guided wave as a function of the height of water. As the height of water increases, $\omega_{c}$ first decreases until $h_{w}=0.3 d$ and then increases again. Given that $\alpha=\sqrt{\left|\omega^{2}-\omega_{c}^{2}\right|} / c$, this number at a fixed frequency first decreases then increases following $\omega_{c}$. The value of the coupling coefficient $\kappa_{22}$ also changes with the water height. Indeed, the pressure distributions shown as insets of Fig. 3(b) show that the modal shape remains relatively stable though the cross-section of the waveguide changes significantly. Owing to the circular symmetry of the waveguide for $h_{w}=0$, the two independent modes $\omega_{0 a}$ and $\omega_{0 b}$ are degenerate. In contrast, the symmetry of the waveguide is broken as soon as $h_{w} \neq 0$, leaving a single mode defining the least evanescent guided wave. Overall, it is obtained numerically that both changes in $\alpha$ and $\kappa_{22}$ combine to lead to a steady increase in the first three locally resonant frequencies $\omega_{0} /(2 \pi)$ as a function of water level, as shown by Fig. 3(c). The locally-resonant frequencies can thus be tuned continuously by adjusting the water level in the waveguide.

Next, we consider a sonic crystal composed of a periodic sequence of five grafted tubes and we observe the change in locally-resonant band gaps as the water level is changed. Locally-resonant band gaps are expected to appear around the resonant frequencies for the single resonator and their width is known to depend on the reflection coefficient on a single resonator ${ }^{19}$. We first compare in Fig. 4 band structures and transmission spectra for $h_{w}=0$ and $h_{w}=d / 2$, obtained both experimentally and numerically. Normalized pressure distribution at labeled points taken at the 0th, 1st, and 2nd local resonance frequencies respectively are shown in Fig. $4\left(a_{4}-b_{4}\right)$. The insets in Fig. 4(a1) and (b1) show the eigenmodes at the frequencies of the lower edge $(\mathrm{L})$ and the upper edge (U) of the 0-th local resonance band gap. It is seen that the pressure distributions at the upper edge extend both in the resonator and in the waveguide. In contrast, the pressure distributions at the lower edge only concentrate inside the resonator. It was checked that the situation is similar for the other two band gaps. As a result, the lower edges of the band gaps remain almost unchanged, since they are not affected by the waveguide characteristics, while the upper edges shift to higher frequencies as water is filled into the waveguide. Overall, band gaps become wider. Experimental transmission curves appear more rounded than those obtained from numerical simulation, which we attribute to losses that are present in the experiment but are not taken into account numerically. Overall, both numerical and experimental transmissions are consistent and indicate that locally-resonant band gaps enlarge when $h_{w}$ is changed from 0 to $d / 2$. The third band gap that was hardly opened for $h_{w}=0$ especially deepens for $h_{w}=d / 2$. This could be attributed to the 

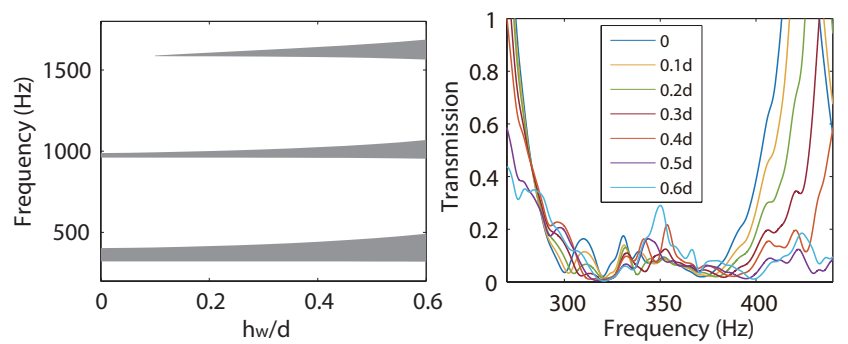

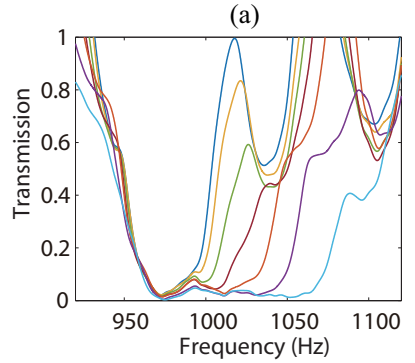

(c)

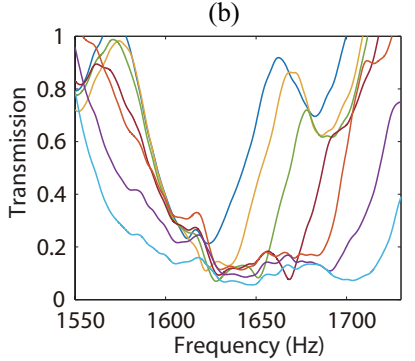

(d)
FIG. 5. Experimental and numerical transmission as a function of water level. (a) The variation of the 0th, 1st and 2nd band gap edges is plotted as a function of the normalized height of water. (b-d) Experimental transmission spectra around the 0th, 1st and 2nd locally resonant band gaps are shown as a function of the height of water.

dispersion shift of certain evanescent bands with changing water level, which were initially closing the third band gap. Experimental and numerical transmissions obtained as a function of water level are reported in Fig. 5. For all 3 band gaps, the lower frequency edge remains almost constant while the upper edge increases continuously. As a result, band gaps become gradually wider as the crosssection of the waveguide decreases with increasing water level. Moreover, the transmission generally gets smaller. Since the level of water in the waveguide can be changed continuously, the width of the band gaps can be tuned accordingly.

In summary, we have demonstrated the tunability of a locally-resonant sonic crystal built using a waveguide grafted with a periodic array of acoustic resonators. Band gaps can be tuned continuously by changing the level of water inside the waveguide, effectively changing the cross-section and thereby the dispersion of evanescent guided waves. Tunability thus does not rely on changing any parameter of the resonators, and neither on changing the propagation velocity of the supporting medium, but instead works by playing with the grafting conditions of the resonators to the propagation matrix. Both the central resonance frequency and the width of the band gaps were shown to depend on the water level. An application could be the acoustic monitoring of liquid level inside tubes and pipes. The present work gives insights for the practical design of tunable acoustic devices. The ideas in this letter can be directly extended to $2 \mathrm{D}$ acoustic systems as well, as well as to the tuning of acoustic metamaterials.

Financial support by the National Natural Science Foundation of China (11702017 and 11532001) is gratefully acknowledged. YFW acknowledges support from Fundamental Research Funds for the Central Universities (2018JBM033 and 2017YJS149). VL acknowledges financial support by the EIPHI Graduate School (ANR17-EURE-0002).

${ }^{1}$ M. S. Kushwaha, P. Halevi, L. Dobrzynski, and B. DjafariRouhani, Phys. Rev. Lett. 71, 2022 (1993).

${ }^{2}$ M. Sigalas, Solid State Commun. 86, 141 (1993).

${ }^{3}$ O. Sigmund and J. S. Jensen, Phil. Trans. R. Soc. Lond. A. 361, 1001 (2003).

${ }^{4}$ Z. Liu, X. Zhang, Y. Mao, Y. Zhu, Z. Yang, C. T. Chan, and P. Sheng, Science 289, 1734 (2000).

${ }^{5}$ W. Feng, Y. Zhang, and W. Che, IEEE. T. Circuits-II 64, 1047 (2017).

${ }^{6}$ T.-T. Wang, Y.-F. Wang, Y.-S. Wang, and V. Laude, Appl. Phys. Lett. 111, 041906 (2017).

${ }^{7}$ M. Oudich, B. Djafari-Rouhani, Y. Pennec, M. B. Assouar, and B. Bonello, J. Appl. Phys. 116, 184504 (2014).

${ }^{8}$ X. Wu, M. Zhang, S. Zuo, H. Huang, and H. Wu, J. Vib. Control p. $1077546318782242(2018)$.

${ }^{9}$ J. S. Jensen, J. Sound Vib. 266, 1053 (2003).

${ }^{10}$ M. I. Hussein, M. J. Leamy, and M. Ruzzene, Appl. Mech. Rev. 66, $040802(2014)$

${ }^{11}$ V. Laude, Phononic crystals: Artificial crystals for sonic, acoustic, and elastic waves (Walter de Gruyter GmbH \& Co KG, Berlin, 2015).

${ }^{12}$ M. Oudich, Y. Li, B. M. Assouar, and Z. Hou, New. J. Phys. 12, 083049 (2010).

${ }^{13}$ O. R. Bilal and M. I. Hussein, Appl. Phys. Lett. 103, 111901 (2013).

${ }^{14}$ M. Chen, D. Meng, H. Zhang, H. Jiang, and Y. Wang, Wave Motion 63, 111 (2016).

${ }^{15}$ A. Khelif, A. Choujaa, S. Benchabane, B. Djafari-Rouhani, and V. Laude, Appl. Phys. Lett. 84, 4400 (2004).

${ }^{16}$ D. P. Elford, L. Chalmers, F. V. Kusmartsev, and G. M. Swallowe, J. Acoust. Soc. Am. 130, 2746 (2011).

${ }^{17}$ Y.-F. Wang and V. Laude, Crystals 7, 323 (2017).

${ }^{18}$ V. Kavungal, G. Farrell, Q. Wu, A. K. Mallik, and Y. Semenova, Opt. Express 26, 8431 (2018).

${ }^{19}$ Y.-F. Wang, V. Laude, and Y.-S. Wang, J. Phys. D. Appl. Phys. 47, 475502 (2014).

${ }^{20}$ V. Laude, Y. Achaoui, S. Benchabane, and A. Khelif, Phys. Rev. B 80, 092301 (2009).

${ }^{21}$ V. Romero-García, J. Sánchez-Pérez, S. Castiñeira-Ibáñez, and L. Garcia-Raffi, Appl. Phys. Lett. 96, 124102 (2010). 